\title{
How Artificial Intelligence can make Competition more Intelligent
}

\author{
Allal MOKEDDEM \\ University of Alger 3, Department of Management and Technology, Algiers, Algeria \\ mokeddem.allal@yahoo.fr
}

Received date: 21 April 2020; Accepted date:21 July 2020; Published date: 4 September 2020

Academic Editor: BOUAMRANE Karim

Copyright @ 2020 . Allal MOKEDDEM. Distributed under Creative Commons Attribution 4.0 International CC-BY 4.0

\begin{abstract}
A company's environment is constantly changing, new competitive models have appeared, new tangible and intangible resources are being built and new competitive regulations form a new competitive framework which opens the field to new opportunities and new threats. With this dynamic, companies are rushing towards the development of their strategic decision system which must be powerful to follow any material (product and service) or immaterial resource (belief, knowledge, know-how) coming from one of the direct competitors. In this context, a conceptual study is carried out with the objective to analyze the evolution of cognition-based competition using artificial intelligence (AI) technology as a support for strategic decision-making; this is in order to transform the competition into an intelligent mode. The finding is presented in an intelligent approach designed to generate automatic competition rules based on deep learning techniques.
\end{abstract}

Keywords: Artificial intelligence (AI), cognitive competition, business model, competitive intelligence.

\section{Introduction}

In a constantly changing competitive environment, the company is faced with the obligation to set up an environmental monitoring system, which measures the innovative strategic actions carried out by the company and its competitors. At this stage, the development of an effective competitive strategy must be consistent with the different dimensions of the strategy, namely structure, leadership, culture and technology.

In the 21st century, the businesses are seen as a center of innovation and wealth creation. In this context, each company is supposed to understand the movements of competitors. Any action is transmitted by the company, in strategic form, such as a

Cite this Article as: Allal MOKEDDEM (2020)," How Artificial Intelligence can make Competition more Intelligent", Communications of the IBIMA, Vol. 2020 (2020), Article ID 622155,

DOI: $10.5171 / 2020.622155$ 
joint venture, or in operational form, such as massive recruitment or downsizing, could be exploited by competitors in order to bring out valuable information.

In a highly competitive environment, it is almost impossible to monitor all kinds of information. For example, competitors' product pages, customer reviews of competing products, product review sites, success stories and customer testimonials, social media channels, scheduled blogs and webinars. All of these resources can also create a wealth of information about the products or marketing resources that your competitors are using.

In this context, according to Alsheibani et al (2020), in-depth analysis techniques serve businesses as a strategic technology for organizations. The world's leading producers of technology, Google, Amazon, IBM, Apple and others have all taken advantage of artificial intelligence (AI) to deliver a better customer experience (Brynjolfsson and Mcafee, 2017), improve productivity (Varian, 2018), and developing commercial collaboration methods (Hunter, 2018).

Faced with this new competitive environment, the rules of competition are constantly evolving. A new environmental state based on cognitive resources which makes it possible to understand this environment. In this article, we analyze the foundations of cognitive competition and how will the competition be intelligent in the era of AI?

This paper is divided into three parts. The first is a staging on the concept of cognitive competition. The second part presents the permanent changes planned in the business plan following the integration of AI. The last part presents how companies can make competition smarter.

\section{Motivation}

The business is looking for the ways to reach a strategic position that ensures its survival and growth. A competitive status that brings opportunities must be reinforced by an effective strategic decision system. In this regard, the companies seeking a position must develop their means of investigation and competitiveness by introducing in-depth analysis methods.

This study opens the doors for Small and medium-sized enterprises to fight in this competition by exploiting their cognitive capacities. The companies using the advanced technological means will be able to dissect each competitive action by highlighting any cognitive resources developed during the competition.

\section{Cognitive Competition: What Is It About?}

Today, the competition is not limited only to managerial or organizational theorems, beyond sociology; social psychology and psychoanalysis also see their importance in the competitive world. In this stage, the company has become a cognitive development entity. The cognition is mainly based on a set of theorems such as the theory of strategy based on resources and competences Penrose (1959); Hamel and Prahalad (1990); Barney (1995), also the organizational learning theorem Argyris and Schön (1978) which emphasizes the cognitive capabilities of organizations.

A competitive environment based on cognition is an environment that transfers not only products and services, but also other resources such as ideologies, perceptions, values and beliefs... In this context, highly competitive companies are looking for ways to take into account the cognitive phenomena of competition. This type of business brings together research and development centers as well as institutional players around innovation projects with regional, national and international vocation (Laroche and Nioche, 2015).

The cognitive competition is measured in terms of the cause and effect relationship between phenomena. At this level, the decision maker analyzes each cognitive action, in order to highlight information that enriches the identification and deliberation phases of the strategy. Contrary to the traditional approach, the cognition seeks to detect what prompts strategic change, not just knowing the strategic purposes. The decision maker can understand the strategy as a set of connected actions which reveals a set of information and knowledge on the 
state of competition which can be converted to competitive opportunities.

A competitive environment brings together all atomic competitors, homogeneous products (Cabral, 2000), incomplete information on the environment and all companies will have equal access to technology and resources. The position in a competitive environment will not happen outside of a unique and distinctive resource base. Certainly, some benefit from unique resources, such as patents, and others can win the competition through strategic actions such as merger, absorption and alliances. In this context, according to Levine et al (2017) two types of cognitive skills are essential to compete (see Figure 1).

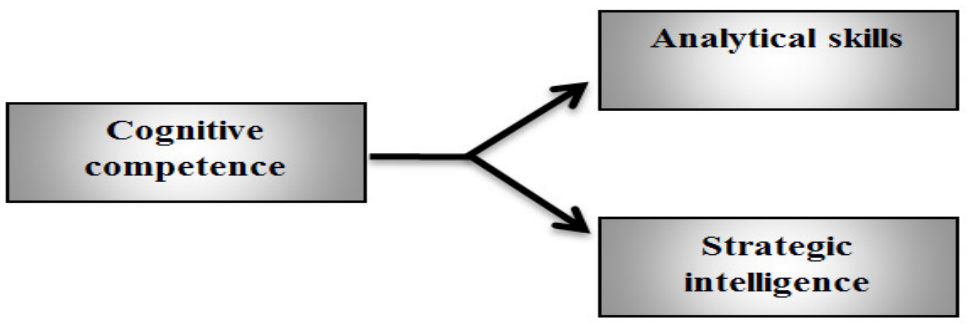

Fig 1. The foundations of cognitive competence

source: Author developed

The competing companies measure their potentials via two types of skills, namely analytical skills defined according to the ability to reason through abstract problems. The second is presented with intelligence strategic resource that allows the company's competitive system to be proactive in the face of any competitive action. In a first plan, the analytical skills carry two dimensions of use. The inside dimension seems likely to make better decisions during the optimization and the coordination between the different activities. This has been confirmed by Helfat and Martin (2015); the companies that have a reservoir of analytical skills are those that can achieve better management and synchronization of their activities. In this context, better management and optimization of the company's value chain are reflected in the control of how and by what means individuals perceive and think during the design and implementation of the strategy. This strategic reasoning is achieved through a set of skills known as social cognition (Frith and Singer, 2008).

The external analysis dimension is used to detect threats and seize opportunities. This possibility is materialized by the dynamic capacities defined by Teece (2007) 'the capacity of the company to integrate, develop and reconfigure its internal and external skills to cope with a rapidly changing environment'. Such an expression can be measured by direct observation of the individual behavior of each actor involved in the competition. This facilitates the environmental analysis process and therefore clearly defines the competitive model that will be adopted by the company.

Strategic intelligence is the second basic resource in a competitive market based on cognition. Strategic intelligence is a set of actions and activities used to find, analyze and evaluate all commercial information in order to achieve a competitive objective (Abd et al, 2019). According to the Intelligence Report (2020), strategic intelligence is designed to provide leaders with critical information to effectively define and adjust strategy. The concept of strategic intelligence is close to other concepts such as economic intelligence and competitive intelligence. According to Huyghe (2010), strategic intelligence is based on methods intended to clarify reality and discover projects and resources in a situation of competition or conflict. On the 
contrary, economic intelligence is based on research, processing, dissemination and protection of information useful to economic actors. In a nutshell, economic intelligence is a subcategory of strategic intelligence. All of these mechanisms make competition smarter and use open source of information management techniques to gain an advantage over the competition.

The strategic intelligence process is based on an effective strategic intelligence system. The company does not only seek to collect information on the competition, but also to create a competitive network based on resources. This is in order to clarify the facts of competition (Levine et al, 2017). The following points illustrate the structure of an industry that uses the strategic intelligence profiling tool during the implementation of the strategy: Bleoju and Capatina (2015)

\section{Intelligence Provider}

The intelligence providers are those who demonstrate their ability to harness strategic intelligence and transfer it into new methods and technological tools. The suppliers who feed the managerial context with new organizational, cultural and human approaches, aim to involve all employees in technical, social and environmental performance. This privilege offers the option to present knowledge in models intended to understand, solve and analyze new environmental situations.

\section{Vigilant Learner}

The learning companies present the second style of companies that contradict spy practices. This type of business is more flexible to deal with new competitive situations. At this level, learning companies are those that constantly challenge their business models, in order to clarify and deepen the overall vision of the organization. In this context, any decision processed becomes a space for sharing and collaboration.

The learning companies can set up collaboration and sharing channels with the various environmental players. The channels are presented by a network which encourages the business actors to engage in the process of developing the competitive environment. In this context, the organizations are also able to react quickly and in a timely manner to a rapidly changing environment. According to Hassan et al (2017), the organizations engage in the search for new knowledge (exploration) in order to ensure current viability, in parallel while devoting our resources to the development of things already known (exploitation). In this regard, learning increases competition via two learning modes, adaptive and generative Luo, (2020); Escrig et al (2019). The first generally increases performance in the form of increased profitability, return on investment and growth in the market share. The second increases reliability by enabling companies to develop sustainable business models, thereby providing products and services that are superior to the competition.

\section{Opportunity Captor}

The third category of companies is presented by companies that capture opportunities. An opportunity is a favourable circumstance. The concept of opportunities brings together a set of companies around different approaches: Loilier and Tellier (2020); Germain, (2010)

\section{- $\quad$ Strategic Approach}

In the Harvard model, the notion of opportunity takes into account any external factor likely to favourably influence the prospects for growth and the trajectory of an organization. In a strategic context, the managers are more sensitive to the search for threats and have a strong feeling of mastery of the environment. The strategic approach makes it possible to analyze the competition by using the competition rule which says: "behind every promising opportunity lies a destructive threat".

\section{- $\quad$ Praxeological Approach}

In the praxeological approach, the discovery and construction of opportunities are integrated with the strategic process. According to Andrews' approach, the opportunity makes it possible to identify strategic and operational actions. These actions make it possible to exploit the 
strategic potential of the organization. Once the business idea is issued, the exploration and evaluation process is displayed.

The process of discovering and building of opportunities is divided into two phases. A phase of reflection focused on determining the goals and general orientation and a more operational phase focused on the seizure of opportunities. The reflection phase is included in the strategy formulation approach which leads to identify the distinctive skills of the company. In this phase, the discovery of opportunities requires a better combination of resources.

\section{- $\quad$ Generating Approach}

The identification of opportunities requires strategic resources that aim either to strengthen the organization's capacity to resist in the face of unprecedented disruptions, or to build the capture of strategic opportunities. In this context, the companies project themselves into an evolving and proactive competitive status. The realization of such an approach consists in making innovation a determining factor of competition. At this stage, the companies define competition according to exogenous and endogenous parameters, for example, the technology which defines the new efficient conditions of production imposed on firm. Likewise, the inspirational approaches indicate that competition is accentuated by the effect of internal factors. For example, the organizational performance, in particular the quality of organizational routines that have been developed to compete in an innovative context.

\section{Opportunity Defender}

The latest style is presented by the protectors of opportunities. At this level, the companies defend their interests against proactive spies. The concept of redirecting competition only functions with a good diagnosis of the internal and external environment. In this context, the actors of opportunity defender use different methods to protect their profits and develop others. First, false alarms distort competition. The companies disclose misleading information about their strategic protocols to competitors in order to contradict the behavior of competitors. The second strategic action very heavily used by protective companies is to invest well in micro niches which require very specific and sometimes scarce resources and means. The third aspect frequently used by companies is to explain part of their business plan in order to create interoperability channels between the operating systems of competitors and that of the company.

In similar circumstances, the competitive environment takes on a more rigorous dimension. In this context, many companies are looking for ways to explore all the information from different data sources by manipulating and directing very precise information on the environment and on the behavior of each actor. But how can businesses succeed in the same competitive environment?

\section{AI For Competitive Purposes}

With the advent of advanced technologies that increase the ability of companies to respond proactively to competition, the strategic and operational system of companies is growing and becoming more complex. A competitive decision-making system is based on technological tools that carry the strategic dimension. The strategic dimension is presented through an analysis of the movements of each player: where, why, what, how and by what means this competitive action will be carried out.

The competitive trends have transformed the competitive methods and protocols adopted by companies. The companies are oriented towards the angle which sees AI as a support for the development of business capacities of the company (Davenport and Ronanki, 2018). In this context, Tripathi and Parmar (2019) indicate that AI projects the company to a higher level of thinking. From a decisional point of view, AI transforms and makes machines, procedure and decisions more intelligent.

\section{AI-Based Business Plans}

According to Wright and Schultz (2018), the recent advances in robotics, artificial intelligence and machine learning allow 
machines to automate activities that once seemed immune to disturbance. These activities based on tasks of thinking, tacit learning, detecting emotions and even deciphering the behavior of competitors. Overall, AI can meet three important managerial needs: automate business processes, obtain competitive information through data analysis, and interact with customers and employees (Davenport and Ronanki, 2018). Generally, the automating activities are a complex task that requires a continuous change in cognitive resource base.
The technology with its different variants is no longer used as a tool for sharing and transferring knowledge, but rather considered as an instrument of cognitive investigation. From an operational tool to a strategic tool that can make the company smarter in managing its business portfolio. A study was conducted with 250 business leaders who knew perfectly how to use AI in businesses. More than half said their main goal was to continually improve the product line to offer the best products to their customers Deloitte (2017). (See Fig 2)

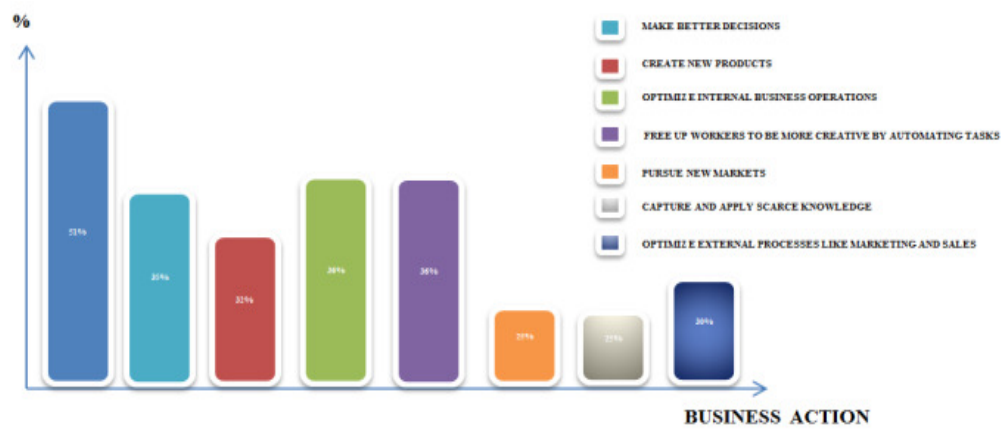

Fig 2. The Business Benefits of AI

Source: Author developed

According to studies conducted by Vinogradova et al (2019), the exploitation of advanced technologies in the business world has contributed to the development of innovative manufacturing approaches. This new situation is the consequence of the development of new trades, new knowledge and new skills that will be increasingly requested by business leaders. In fact, according to Global Revenue for Enterprise Applications (2019), the annual revenues from the use of artificial intelligence applications will increase from $\$ 7.6$ billion in 2018 to $\$ 107.3$ billion in 2025 and by 2030 , the global economy could reach $\$ 15.7$ trillion.

All are based on information; the design of an intelligent industrial system is a process which not only includes the production procedure with its possible requirements such as quality standards, production measurements, flow measurement, logistics flow and capital flow ... etc, but requires an automatic detection of each change affecting competition, collaboration, learning, analysis, cognition, decisionmaking and control of the decisions process.

According to Li et al (2017), AI technology, presented by deep learning techniques, automatic natural language processing, partitioning and automatic classification, learning machines, pattern recognition and predictive techniques ... etc., has changed the concept and the way by which a business can be classified as a competitive business. A business model refers to a set of interdependent activities covering the whole of the company (Gassmann et al, 2017). A business model will never be fully explained to the public. Leaders are looking for the ways to innovate and differentiate themselves from the competition. An innovative multidisciplinary business model is one that creates significant change in strategy, organization, culture and leadership. The manifested interest in AI has been underscored by the business community. The study conducted by Columbus (2017) with more than 3000 interviews with business leaders. As a 
result, the study found that $84 \%$ believe that AI will allow the company to acquire and maintain its competitive advantage and $75 \%$ said that AI will have access to new business concepts. In this context, we need to think about how AI could invent a distinctive business model.

The development of advanced technologies has opened up commercial channels that can act anywhere with anyone, anything, anytime and on any medium, be it physical, digital or virtual (Lindgren and Rasmussen, 2016). Indeed, the companies must constantly know how their business plan works and how is it built? In this part, we travel in the world of AI and we analyze how to do and act when advanced technological tools are integrated into the strategic system of the company.

First, the companies need to familiarize themselves with the language of the business world in order to feel in harmony with the expected technological developments (Lindgren, 2017). In this regard, Valter et al (2018) present a conceptual model that examines the relationship that connects four integral parts, namely: the human intermediary; the machine intermediary; analysis of business models and the business plan ecosystem.

The development of a business model is a mission by a thousand miles. First, the human action which brings together any interaction that joins the business reality to the business model (Valter et al, 2018). The business model is a perceptual representation of business reality. The human factor updates the model with his understanding how things act in business reality. This interpretation will never be put outside the business environment. The model has undergone interactions that generate a new knowledge from different areas of expertise. This situation opens the door to new styles of potential understanding that offer opportunities for exploring cross-border knowledge.

The technology slides provide opportunities during developing a transparent and effective business plan. A machine presented by data entry robots, heat maps...etc, which continuously observes the reality of business. We cite devices that analyze customer behavior via machines installed in sales areas. In this context, a business model triggers one or more events on the intermediate machine. If a business owner wants to have a number of customers in his store within a specific period of time, then this event triggers a business model that at one time treats a limited number of customers in the store. The business model considers price as a determining factor, contributing to the decrease or increase in the prices of specialized offers displayed via automatic display panels.

The third impact factor describes the relationship between artificial intelligence via deep learning models that reflect the intersection between the human factor, the machine and the desired business plan. The AI and deep learning algorithms analyze the ecosystems of business models in order to optimize existing business models and suggest new models based on the success of others that operate in various competitive contexts. The deep learning algorithms offer the possibility of building improved business models using an artificial intelligence tool. For this purpose, each design phase corresponds to a particular state of competition. Consequently, the deep learning algorithms transform business plans into hierarchical structures. For example, the lowest level consisted only of lower-class customers versus the higher levels reserved for the most important customers in terms of quantity of purchases and who require specific negotiation approaches.

In the section below, we describe perfectly how the company can transform the competition and make it more intelligent.

\section{How to Make Competition More Intelligent?}

The company is looking for a way to strengthen its competitiveness through better quality provided, better delivery times and greater flexibility in adjusting costs. In this context, the development of an effective operational strategy that reflects the planning, design and implementation of strategic decisions is the only path to the development of a distinctive competitive status. At this level, the analysis of the 
competitor's behavior offers a precious opportunity to develop a competitive intelligence system. This system is based on a strategic method which constantly anticipates the movements of competitors (Guo et al, 2017).

Competitive analysis is powered by advanced technologies allowing efficient access and analysis of large data sets. According to Kraus et al (2020), the deep learning and deep neural networks promise to maintain improvements, performance and make better predictions compared to traditional methods. However, the neural networks have changed the way we compete in a market. Accordingly, hundreds of layers and millions of complex neurons (He et al, 2016) hide sophisticated information and resources about the competition.

The deep learning has not only been very successful in natural language processing, but also in image classification, object detection, object localization and image generation (Kraus et al, 2020). As a result, large companies around the world like Google in 2016 launched an update to their translation system that uses deep learning to improve the translation accuracy that accompanies the human performance approach. Moreover, Amazon and Netflix use deep neural networks in their recommendation system. In this respect, according to Sassi et al (2016) each competitive action carried out receives treatment based on the following competitive intelligence approach:

\section{Collection of Actions}

The competitive actions are made by the competition. Its actions are reflected in the company's decision-making system. Often, the company's strategic decision-making system is encrypted, which makes the collection process quite difficult. In this context, the identification of the strategic source of information requires fairly powerful means of collection. The WebRadar is a powerful tool that offers companies the ability to follow market trends. For example, we cite the WebRadar that assesses the reputation of the company. Several advantages are presented: I) it discovers the influences that impact the reputation of the company; II) it monitors your image in real time and III) analyzes your image with the public. Especially since, according to Chaveesuk et al (2019), in the digital age, companies leave behind so much information regarding their online activities such as research, purchases and reading articles, etc. These pieces of information are produced daily and used by companies for commercial purposes.

For this reason, several applications have been developed to provide information and news about the competition. Adthena (2018) continuously monitors millions of keywords around the world. This technology allows you to see on what terms your competitors are bidding and the resulting impact on your campaigns. With Adthena (2018) Greenlight was able to instantly analyze the performance of competitors via a device that quickly scans advertising copies, a task that used to take hours and days. Therefore, by eliminating these delays, Greenlight was able to provide information to customers earlier and spend more time in the design and execution of their plan.

\section{Actions Association}

The actions collected must be grouped in such a way that the reactions of competitors to the launched offer are in the same cluster. The competitors' reactions focus on internal and external competitive criteria. We cite the cluster that combines both internal and external factors, namely the style of marketing communication and the level of quality. The companies are oriented towards the use of a brand communication method that disseminates imaginary concepts about the product. This creates specificity in the use of this product and a difference in its brand and its nature.

\section{Rule Generation}

This step is reserved for the generation of competition rules in the form of an algorithm (if... .. then). In this respect, the rough set theory which can be considered as an intelligent mathematical tool offers the option of extracting and defining competitive rules from the observation of approximate and inaccurate data. The rough set theory is applied to generate rules 
and predict the actions of competitors based on the history of the competition (see

Fig 3).

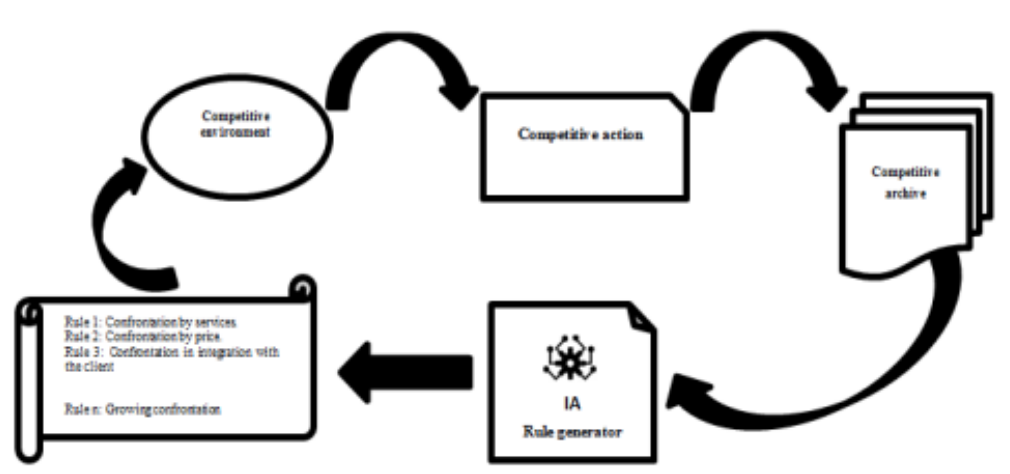

Fig 3. The system generating the competition rules

source: Author developed

The generation of a competition rule is subject to an algorithm which instantly connects to the competitive archive store. As an example, we simulate the rule of "confrontation by services" in a telecommunications market structured by a set of companies (C1, C2 ... C8). The system generator of competition rules acts according to the algorithm presented below:

Rule 1: A1: If (actor $=$ Concurrent 1 ) and (actor $=$ Concurrent 2) and (Type of service $=2 \mathrm{Go}$ communication bonus line) and (target $=$ Actual customer, Prepaid line) then (Owen company will react with action A5 and A8 respectively)

\section{Conclusion}

The competition is defined by a set of actions presented in a business plan. The Knowing of the state of competition is the unique way to develop an effective competitive system. At this stage, the cognition and the perception of competition have been accompanied by the development of methods and big data techniques analysis. In this regard, the combination between the human factor (thinking), machine (power) and the business plan ecosystem (environment) presents the three basic foundations for the development of the intelligent competitive system. The latter is put into operation as soon as a competitive action appears. This action is the subject of an advanced strategic analysis in order to generate new competition rules.

\section{References}

- Alsheibani, S., Messom, C. and Cheung, Y. (2020), 'Re-thinking the Competitive Landscape of Artificial Intelligence' In Proceedings of the 53rd Hawaii International Conference on System Sciences.

- $\quad$ Abd, KMA., Abbas, SA. and Khudair, AH. (2019), 'Impact of Strategic Management Practices on Organizational Entrepreneurship Mediating Effect of Strategic Intelligence,' Academy of Strategic Management Journal.

- $\quad$ Argyris, C and Schön, D. (1978), 'What is an organization that it may learn,' Organizational learning: A theory perspective, Argyris C, Schoen DA (eds). Addison-Wesley: Reading, MA, 8-29.

- Adthena., (2018), "Every competitor, every move - let machine learning give you the edge in your market, ' [Online], 
[Retrieved April 11, 2020], https://www.adthena.com/under-thehood/

- Adthena., (2018), "With 90\% higher productivity, Greenlight can give $110 \%$ to clients, ' [Online], [Retrieved April 12, 2020], https://www.adthena.com/wpcontent/uploads/2019/11/GreenlightCase-Study.pdf

- Brynjolfsson, E and Mcafee, A. (2017), 'The business of artificial intelligence,' Harvard Business Review, 1-20.

- Bleoju, G and Capatina, A. (2015), 'Leveraging organizational knowledge vision through Strategic Intelligence profiling-the case of the Romanian software industry,' Journal of Intelligence Studies in Business, 5(2).

- $\quad$ Barney, JB. (1995) 'Looking inside for competitive advantage,' Academy of Management Perspectives, 9(4), 49-61.

- Cabral, LMB. (2000) 'Introduction to industrial organization,' Cambridge, Massachusetts: MIT Press.

- Columbus, L. (2017), "How Artificial Intelligence is Revolutionizing Business in 2017, ' [Online], [Retrieved May 3, 2020], https://www.forbes.com/sites/louiscolum bus/2017/09/10/how-artificial-

intelligence-is-revolutionizing-business-in2017/\#11ba98b15463

- Chaveesuk, S., Khalid, B. and Chaiyasoonthorn, W. (2019), 'Emergence of New Business Environment with Big Data and Artificial Intelligence' In Proceedings of the 9th International Conference on Information Communication and Management (pp. 181-185).

- $\quad$ Davenport, TH and Ronanki, R. (2018), 'Artificial intelligence for the real world,' Harvard business review, 96(1), 108-116.

- Deloitte.I., (2017), " State of AI in the Enterprise, ' 2nd Edition. [Online], [Retrieved April 10, 2020], https://www2.deloitte.com/content/dam/ insights/us/articles/4780_State-of-AI-inthe-enterprise/DI_State-of-AI-in-theenterprise-2nd-ed.pdf
- $\quad$ Escrig, ED., Broch, FFM., Alcamí, RL. and Gómez, RC. (2019), 'How to enhance radical innovation? The importance of organizational design and generative learning,' Review of Managerial Science, 122.

- Frith, CD and Singer, T. (2008), 'The role of social cognition in decision making,' Philosophical Transactions of the Royal Society B: Biological Sciences, 363(1511), 3875-3886.

- Global Revenue for Enterprise Applications., (2019), " Global Revenue for Enterprise Applications is Expected to Reach \$107.3 Billion by 2025, as Enterprises Move From Trials to Deployments, According to Tractica, [Online], [Retrieved April 06, 2020], https://www.businesswire.com/news/ho me/20191014005213/en/GlobalRevenue-Enterprise-ApplicationsExpected-Reach-107.3

- Gassmann, O., Frankenberger, K. and Sauer, R. (2017), 'A primer on theoretically exploring the field of business model innovation, ' 45-48.

- Guo, L., Sharma, R., Yin, L., Lu, R. and Rong, K. (2017), 'Automated competitor analysis using big data analytics, ' Business process management journal.

- Germain, O. (2010) 'Quand l'opportunité rencontre la stratégie,' Revue française de gestion, (7), 171-187.

- He, K., Zhang, X., Ren, S. and Sun, J. (2016), 'Deep residual learning for image recognition' In Proceedings of the IEEE conference on computer vision and pattern recognition, (pp. 770-778).

- Hamel, G and Prahalad, CK. (1990), 'The core competence of the corporation,' Harvard business review, 68(3), 79-91.

- Huyghe, FB. (2010) 'Information, pouvoir et usage: l'infostratégie,' Huyghe. fr,(blog de l'auteur), https://www. huyghe. fr/actu_321. htm.

- Huyghe, FB. (2012) 'Information, pouvoir et usage : l'infostratégie > Intelligence économique : du savoir à 
l'influence,' Huyghe. fr,(blog de l'auteur), https://www.huyghe.fr/actu_283.htm.

- Hassan, S., Iqbal, YMJ. and Ghias, W. (2017), 'Organizational Learning and Competitive Advantage in Banking Sector of Pakistan,' Global Social Sciences Review, 2(1), 79-90.

- Helfat, CE and Martin, JA. (2015), 'Dynamic managerial capabilities: Review and assessment of managerial impact on strategic change, ' Journal of management, 41(5), 1281-1312.

- Hunter, J. (2018) 'Cover story: Artificial intelligence in school education: Are you ready for it?', Education Technology Solutions, (85), 28.

- Intelligence, WIS. (2020) 'Strategic Intelligence,' Intelligence in the National Security Enterprise: An Introduction, 111.

- $\quad$ Kraus, M., Feuerriegel, S. and Oztekin, A. (2020), 'Deep learning in business analytics and operations research: Models, applications and managerial implications,' European Journal of Operational Research, 281(3), 628-641.

- $\quad$ Li, BH., Hou, BC., Yu, WT., Lu, XB. and Yang, CW. (2017), 'Applications of artificial intelligence in intelligent manufacturing: a review,' Frontiers of Information Technology \& Electronic Engineering, 18(1), 86-96.

- $\quad$ Luo, FL. (2020) 'Adaptive Learning for Symbol Detection,' 197-211.

- Loilier, T and Tellier, A. (2020), 'Les grands auteurs en stratégie,' Éditions EMS.

- Laroche, H and Nioche, JP. (2015), 'L'approche cognitive de la stratégie d'entreprise,' Revue française de gestion, 41(253), 97-120.

- Lindgren, $\mathrm{P}$ and Rasmussen, $\mathrm{OH}$. (2016), 'The business model ecosystem,' Journal of Multi BMI, 4, 1.

- $\quad$ Lindgren, P. (2017) 'Advanced business model innovation,' Wireless Personal Communications, 95(1), 127-144.

- Levine, SS., Bernard, M. and Nagel, R. (2017), 'Strategic intelligence: The cognitive capability to anticipate competitor behavior,' Strategic Management Journal, 38(12), 2390-2423

- Penrose, E. (1959) 'The theory of the growth of the firm,' Oxford university press: Blackwell.

- Sassi, DB., Frini, A., Karaa, WBA. and Kraiem, N. (2016), 'A Competitive Intelligence Solution to Predict Competitor Action Using K-modes Algorithm and Rough Set Theory,' In KES, (pp. 597-606).

- $\quad$ Teece, DJ. (2007) 'Explicating dynamic capabilities: the nature and microfoundations of (sustainable) enterprise performance,' Strategic management journal, 28(13), 1319-1350.

- Tripathi, A and Parmar, R. (2019), 'Business reshaping using Artificial Intelligence, '

- Varian, H. (2018) 'Artificial intelligence, economics, and industrial organization,' National Bureau of Economic Research, (No. w24839).

- Vinogradova, AI., Fomina, YV., Gorodischeva, AN., Astapenko, EV. and Bedareva, AV. (2019), 'Artificial intelligence capabilities classification in business environment,' In Journal of Physics, Conference Series (Vol. 1399, No. 3, p. 033098). IOP Publishing.

- Valter, P., Lindgren, P. and Prasad, R. (2018), 'Advanced business model innovation supported by artificial intelligence and deep learning,' Wireless Personal Communications, 100(1), 97-111.

- Valter, P., Lindgren, P. and Prasad, R. (2018), 'The consequences of artificial intelligence and deep learning in a world of persuasive business models,' IEEE Aerospace and Electronic Systems Magazine, 33(5-6), 80-88.

- Wright, SA and Schultz, AE. (2018), 'The rising tide of artificial intelligence and business automation: Developing an ethical framework,' Business Horizons, 61(6), 823832. 